\title{
User-centric cognitive assessment. Evaluation of attention in special working centres: from paper to Kinect.
}

\author{
Vilaro, A. \& Orero, P. \\ Centre d'Accessibilitat i Intel-ligència Ambiental de Catalunya, CAIAC. \\ Universitat Autònoma de Barcelona, UAB.
}

KEYWORD

Cognitive deterioration

Special working centres

Cognitive evaluation

Attention

Kinect

\section{Introduction}

The employment of cognitive impaired people in special working centres contributes to improve their quality of life and provides many benefits such as social integration. The increase in life expectancy raises a new issue in the context of special working centres: cognitive deterioration of the elder mentally disabled people associated with premature ageing. This deterioration is usually related to difficulties in maintaining attention and problems associated with memory, plus many other physical related changes.

Special working centres need to evaluate the physical and cognitive state of their workers when they join the workplace for the first time in order to guarantee that the job matches the worker's profile [PAREDES GASCÓN, M. et al. 2012]. Moreover, there is a need to have a longitudinal assessment throughout the working life in order to ensure that the employee retains the skills that were described in the initial evaluation. For example, the inability to maintain sustained attention (concentration) can hamper the worker to complete the tasks or steps in a process; or problems with memory may difficult remembering already learned tasks or learning new ones. Thus, the deterioration may hinder the performance of job duties or even stay in employment. When possible cases of cognitive impairment are detected in the working place, and evaluation is required by specialized staff (mainly psychologists) in order to provide information to help decide whether he/she should retire. The evaluation is made through questionnaires and interviews, procedure that usually takes a long time. Furthermore, the absence of a history recording that objectively shows the evolution of 
cognitive deterioration makes it difficult to evidence the changes to the public administration. Special working centres require a solution to optimize the evaluation process.

In order to address this need, PROLOG project aims to provide a complementary tool for the evaluation. The project develops an ICT tool with a set of tests to assess cognitive deterioration of workers with cognitive impairments. PROLOG has its innovation in incorporating Kinect technology that recognizes body movements and voice to interact with applications. This technology, as applied to the field of rehabilitation of disabled people, is being used for the first time to develop tests to assess attentional capacity. The project also provides a platform to record the execution of various tasks and users and their carers can see the evolution over time in order to detect possible cognitive impairment.

The project present three important improvements regarding the current evaluation tools: 1) Creation of new interaction of existing attentional tests which are already in use for evaluation of workers with cognitive disabilities; 2) Creation of evaluation benchmarking to correlate the results of attentional tests. Values will be objective and numeric, allowing a longitudinal evaluation across the working life of the person; and 3) Creation of personalised exercises, complementary with tests, to exercise attention and maintain skills.

\section{Methodology}

A series of tests were designed to evaluate attention. Attention was chosen because it is a fundamental cognitive function that is usually seen rapidly affected in the presence of cognitive deterioration.

We aim to create attractive and motivating tasks, using similar stimuli and goals presented in videogames. During the first year, three tests are being designed to evaluate different dimensions of attention: specifically, sustained attention and selective attention. The proposed tests are based on existing and validated psychological tests.
The tests design and the platform functionalities are presented below. At the end of the section the validation process for the tests is presented.

\subsection{Evaluating sustained attention}

Sustained attention can be defined as the ability to maintain attention during continuous and repetitive activity. We designed two tests aimed at assessing the ability to sustain attention over a period of time. For example, the quality control of a production line requires the ability to sustain attention. There are several tests to measure it: Cancellation tasks (letters or figures), reaction time tasks, CPT (Continuous Performance Test), DVT (Digit Vigilance Test), TAP (Test of Attentional Performance), or SART (Sustained Attention to Response), among others.

We adapted the test SART (Sustained Attention to Response [Robertson et al., 1997; Manly et al., 1999] measures the ability to inhibit responses to infrequent and unpredictable stimuli during a period of rhythmic and rapid responses to a frequent stimuli (generally associated with the detection of an unlikely-to-occur stimuli). In the SART test one digit at a time is presented, and the participant's task is to quickly respond to all digits except to one in particular (e.g. touching the screen in every trial, except when the number 3 is presented). Thus, it is a reaction time task that evaluates sustained attention through an element of response suppression.

In our adaptation of the test we replaced the digits for images (images of insects, for example) in order to make it more attractive to users. The goal of the game would be to hunt insects in a forest scenario. During the test, images of insects appear rhythmically in various predetermined positions of the screen. Similar to SART test, the task is to "catch" all the insects, except one in particular. To perform the task, the user has to perform a motor response (i.e. raise the hand simulating the action of grasping) when the stimuli is detected, or do nothing when appropriate. Thus, the user has to pay attention to avoid hunting a pre-defined insect or element. The test finishes when the participant exceeds a determined number of errors, or when a time limit is over. After the test, performance results 
are presented such as for how long the test lasted or the number of errors during a predefined interval of time.

The other designed test to measure sustained attention is based on the TAP test [Zimmermann, et al., 2002]. This test requires the comparison of two subsequent stimuli in order to determine if these two stimuli have a predetermined feature in common. This procedure requires the use of working memory and also mental flexibility. In a more complex variant, it includes the ability to divide attention, as two aspects of the stimulus must be taken into account.

In our adaptation, users were presented with a table countertop where different-shaped wood figures may appear. Following the example of the insects, the user's task is to detect as quickly as possible whether the stimulus is the same as before (e.g. same wooden-figure) or not. Thus, there are two possible answers (same or different) that the user should indicate raising the right or left hand in front of Kinect in order to activate buttons showed in the screen. When the tests finishes, results such as the percentage of correct answers, errors and omissions, as well as reaction time are presented.

\subsection{Evaluating selective attention}

Selective attention is defined as the ability to attend one or two important stimuli, while deliberately suppressing the consciousness of the distracting stimuli. In order to assess selective attention we adapted the Flanker Compatibility Effect [Eriksen, 1974]. This is an experimental task designed to study factors that may affect selective attention, and to what extent information processing of irrelevant information occurs. The experimental task is to attend a central stimulus flanked by other stimuli called flanks or distracters. The subject's task is to identify the central stimulus (for example, a letter) and ignore the side letters.

In our adaptation of the test the stimuli are images of animals that point to a particular direction (for example, images of three caterpillars pointing to the left). User task's is to say whether the central insect looks to the left or right (caterpillars presented on the sides can

Special Issue \#7

http://adcaij.usal.es

ISSN: 2255/2863

DOI: http://dx.doi.ort/10.14201ADECAIJ2013171922 point to the same direction or the opposite). The user must indicate if the direction of the track is to the right or left. At the end of the test performance results would inform about percentages of correct responses, errors and omissions, as well as the average reaction time.

\subsection{The platform}

The tests are implemented on a platform that allows the management of both, user information and tests parameters. The hardware architecture of the system is based on a Windows PC with a Kinect camera. The platform will include a local database to store data of different users.

User profiles will include personal information (i.e. age, gender, diagnosis, workplace, etc.), as well as the history recording of the tests results. Specifically, the platform will display the results of the tests carried out by the users and the evolution over time by means of tables and graphs. Results can be displayed for each particular session, or in graphical comparisons between multiple sessions.

Also, given the user centric approach of the application, the test parameters can be modified through a configuration screen before starting, and these parameters can be saved for each user in the platform in order to adapt the difficulty of the tests to different users. For example, it may offer the possibility to choose a category of stimuli (fruits, colours, shapes, etc.), or to define which particular element is defined as target stimulus within a category. Additionally, other parameters may be configured such as presentation time of the stimuli, the duration of the interval between stimuli, or the number of trials in each test.

In a future phase of the project it is expected to provide support for a protocol for special working centres aimed at evaluating the continuity in the workplace.

\subsection{Validation process}


Once the tests are implemented we will conduct a validation of the tests. The tests will be run in several special working centres in Catalonia. Also, results for non mentally disabled people will be obtained.

Two groups of cognitive impaired users will be defined in order to compare tests results, one group showing signs of cognitive deterioration, and a second group with no such signs. Any other variables that might affect the results will be taken into account. Also, in order to verify the concurrent validity of the proposed tests, the results of both groups will be compared with other existing tests and evaluations (e.g. ICAP test, medical evaluations, etc.).

\section{Conclusion}

It is expected that the PROLOG evaluation tool will have an important impact in several contexts. Health and safety risks will be monitored, and when an employer is no longer able to work, it will be possible to show the progression in the deterioration, avoiding the existing reality of disabled workers having to work until the retirement age of 67 , unable to perform any task, still having to go to work every day.

In the future it is planned to extend the number of tests to assess other cognitive skills such as memory or executive functions. Also, new exercises with Kinect will be created as training sessions to improve or maintain cognitive abilities. Thus, the evaluation tool would be useful to the users themselves (to see their evolution), the company-employer, and finally the government.

\section{Acknowledgment}

The PROLOG project is being developed by the Centre d'Accessibilitat i Intel-ligència Ambiental de Catalunya (CAIAC), together with Indra, Adecco Foundation, PRODIS and UAB, and is part of the Cátedra INDRA de Tecnologías Accesibles.

\section{$5 \quad$ References}

[ERIKSEN, B.A. et al. 1974]

[MANLY, T. et al. 1999]

[PAREDES GASCÓN, M. et al. 2012]

[ROBERTSON, I. H. et al. , 1997]

[ZIMMERMANN, P. et al. , 2002]
ERIKSEN, B. A., \& Eriksen, C. W. (1974). Effects of noise letters upon the identification of a target letter in a nonsearch task. Perception and Psychophysics, 16(1): 143-149.

MANLY, T., Robertson, I.H., Galloway, M., Hawkins, K. (1999). The absent mind: further investigations of sustained attention to response. Neuropsychologia, 37(6): $661-70$.

PAREDES GASCÓN, M., Fernández-Cid Fernández M. \& Ruiz Figueroa, M. J. (2012). Prevención de riesgos laborales entre las personas con discapacidad intelectual en los centros especiales de empleo. Cuadernos de Trabajo Social, 25 (1): 249-260.

ROBERTSON, I. H.; Manly, T. et al. (1997). 'Oops!': performance correlates of every-day attentional failures in traumatic brain injured and normal subjects. Neuropsychologia 35(6): 747-758.

ZIMMERMANN, P., Gondan, M., \& Fimm, B. (2002). KITAP, Testbatterie zur Aufmerksamkeitsprüfung für Kinder [Attention test battery for children]. Herzogenrath, Germany: Vera Fimm, Psychologische Testsysteme. 